\title{
Fission cross sections measured at nTOF with a coincidence method
}

\author{
L. Tassan-Got*, L. Audouin, B. Berthier, L. Ferrant, S. Isaev, C. Le Naour, \\ C. Stéphan, D. Trubert \\ IPN-Orsay, France \\ E-mail: tassango@ipno.in2p3.fr
}

\section{Duran, C. Paradela}

Universidad de Santiago de Compostela, Spain

n-TOF collaboration, CERN

\begin{abstract}
The n-TOF facility at CERN delivers neutrons from $1 \mathrm{eV}$ to $1 \mathrm{GeV}$. Fission events have been characterized by detecting the two fission fragments in coincidence with PPACs (Parallel Plate Avalanche Counters), which allows a clear signature of fission among other reactions occurring at high energy. Two fission detectors $20 \times 20 \mathrm{~cm}^{2}$ in size were placed on each side of the $8 \mathrm{~cm}$ in diameter targets deposited on a thin aluminium backing. To monitor the neutron beam the well known fission cross section of ${ }^{235} \mathrm{U}$ has been used. PPACs are well suited since they can be thin enough not to absorb neutrons. They produce a very fast signal convenient to minimize pile up, and they are equipped for $\mathrm{X}$ and $\mathrm{Y}$ position determination, allowing to obtain a precise map of the $8 \mathrm{~cm}$ in diameter neutron beam. An illustration of the technique will be given for the fission cross sections of ${ }^{234} \mathrm{U}$ and ${ }^{237} \mathrm{~Np}$ from $1 \mathrm{eV}$ to $1 \mathrm{GeV}$.
\end{abstract}

International Workshop on Fast Nuclear Data and Applications - FNDA2006,

April 3-6,2006,

University of Cape Town, South Africa

*Speaker. 


\section{Contents}

1. Introduction 2

2. The n-TOF facility 2

$\begin{array}{lll}\text { 3. The detection system } & 3\end{array}$

4. Signal treatment 4

5. Results 5

6. Conclusion 6

\section{Introduction}

The foreseen possibility of incinerating nuclear wastes requires reactors loaded with higher concentration of minor actinides. In such conditions there is a need of a better accuracy on their neutron cross sections than presently achieved. In addition if reactors based on the thorium cycle are to be developed, the neutronic properties of the main isotopes involved in this cycle should also be known at a better accuracy. We measured the fission cross section of ${ }^{237} \mathrm{~Np}$ which is a possible candidate for incineration, and ${ }^{232} \mathrm{Th},{ }^{233} \mathrm{U},{ }^{234} \mathrm{U}$ which are major isotopes in the thorium cycle.

An accelerator driven system (ADS) is a possible and efficient reactor for incineration of long lived actinides. In such reactors the fuel assembly can suffer high energy tails of the neutron flux. This implies that the fission cross sections should be measured in a large energy range up to the spallation domain.

In a measurement condition, at high energy above $20 \mathrm{MeV}$, neutron collisions can produce not only fission, but also evaporation residues from the target actinide and from lighter elements contained in target backings and electrodes of detectors.Those residual nuclei recoil due to momentum transfer, and they produce signals which can be wrongly ascribed to low amplitude fission signals. Detecting the two fragments in coincidence with PPACs (parallel plate avalanche counters) allows a clean selection of the fission reactions.

\section{The n-TOF facility}

The n-TOF facility at CERN is a pulsed neutron source based on spallation of $20 \mathrm{GeV}$ protons on a lead target[1]. A fraction of the produced neutrons enters a long pipe and the beam is shaped with a collimator system. The long flight path $(185 \mathrm{~m})$ allows an accurate time of flight measurement due to the short spilling time of the protons: $7 \mathrm{~ns}$. The main characteristics of the facility is the large energy range of the neutron flux, from thermal region to a few $\mathrm{GeV}$, and the tremendous instantaneous flux with a low duty cycle. This last aspect is fully exploited in fission by using flash analog to digital converters (FADC) which allow pile up resolution with limitation of data storage for radioactive targets. 


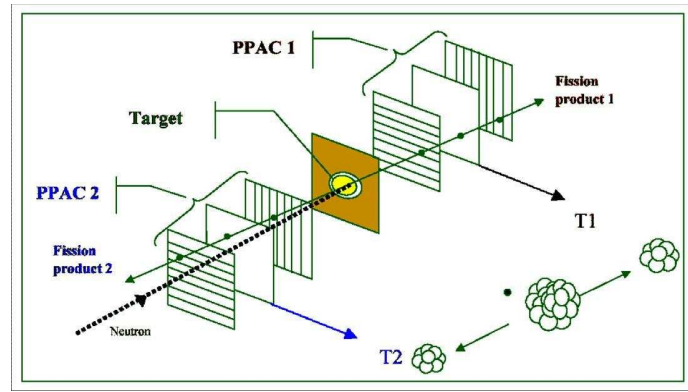

Figure 1: Detection of the 2 fission fragments by 2 PPACs enclosing the target. The $\mathrm{X}$ and $\mathrm{Y}$ localisation in each detector allows a geometrical reconstruction of the fission event.

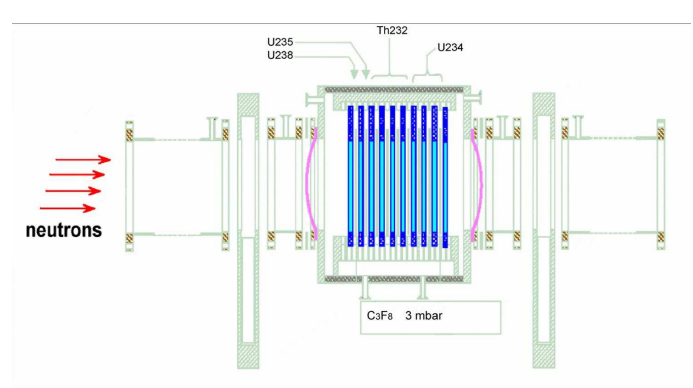

Figure 2: Stack of detectors and targets : 10 detectors interleaved with 9 targets.

\section{The detection system}

The fission fragments are detected in coincidence by 2 PPACs enclosing the emitting target as sketched in figure 1 . The entire system is filled with $\mathrm{C}_{3} \mathrm{~F}_{8}$ gas at 3 mbar which is ionized along the fission path. A bidimensional localisation is achieved on each counter by dividing the cathodes in $2 \mathrm{~mm}$ wide strips which are connected to the intermediate points of a delay line. The localisation is derived from the delays of the signals collected from the two ends of the delay line, as respect to the fast anode signal. Using the back to back characteristic of fission at low energy the two positions allow a full determination of the emitting point on the target and the fission angle. Although the folding angle becomes lower than $180^{\circ}$ at high energy (above $20 \mathrm{MeV}$ ), due to the recoil imparted by the momentum transfer, the above approximation is still accurate for the determination of the fission trajectory.

The full setup is made of a stack of 9 targets interleaved with 10 detectors as shown in figure 2 . Two targets are made of ${ }^{235} \mathrm{U}$ and ${ }^{238} \mathrm{U}$ to determine the neutron flux and its energy dependence. More precisely the setup does not give access to absolute cross sections but to fission cross section ratios between targets. Therefore to derive absolute cross sections one has to know the cross section of a reference isotope. We used ${ }^{235} \mathrm{U}$ and ${ }^{238} \mathrm{U}$ with cross sections taken from ENDF-B6 up to $20 \mathrm{MeV}$, JENDL-HE [2] between 20 and $200 \mathrm{MeV}$, and a constant extrapolation beyond this energy: $1.42 \mathrm{~b}$ for ${ }^{235} \mathrm{U}$ and $1.28 \mathrm{~b}$ for ${ }^{238} \mathrm{U}$.

The request of detecting both fission fragments imposes severe constraints on the thickness of materials. In particular the electrodes of PPACs, the target layers and target backings have to be as thin as possible. Each electrode is made of a $1.5 \mu$ m mylar coated with $50 \mu \mathrm{g} / \mathrm{cm}^{2}$ aluminium as continuous planes for anodes and strips for cathodes. The targets consist of a layer of actinide oxide $\left(0.3 \mathrm{mg} / \mathrm{cm}^{2}\right.$ thickness of actinide $)$ electrodeposited as a disk of $8 \mathrm{~cm}$ in diameter, on a thin aluminium foil, $2 \mu \mathrm{m}$ thick [3]. Overall the total amount of material is $2.7 \mathrm{mg} / \mathrm{cm}^{2}$ of actinide, $6 \mathrm{mg} / \mathrm{cm}^{2}$ of mylar and $7 \mathrm{mg} / \mathrm{cm}^{2}$ of aluminium. In addition two thick windows of $125 \mu \mathrm{m}$ of kapton are inserted for safety reasons. The total material thickness is small enough to avoid any self shielding correction or beam attenuation, and the fraction of scattered neutrons is very small [4].

For trajectories orthogonal to the targets and detectors the fragments always reach the two detecting gaps of each surrounding detector, and the detection efficiency is 1 . For large angles 
corresponding to tilted trajectories, the thickness of crossed materials increases and some fission fragments are stopped before reaching the second detecting gap, especially when the heavy fragment flies through the target backing [5]. In this case the detection efficiency drops below 1 . This effects becomes important beyond $45^{\circ}$. Contrary to the case of cross section measurements with ionisation chambers where a full angular efficiency is assumed, we know in our case that this efficiency is reduced, but we measure the fission angle to correct for the efficiency drop.

\section{Signal treatment}

Each detector delivers 5 signals: 1 fast anode signal and 4 signals from the ends of the two delay lines. The 50 signals are recorded by FADCs running at $500 \mathrm{Ms} / \mathrm{s}$.

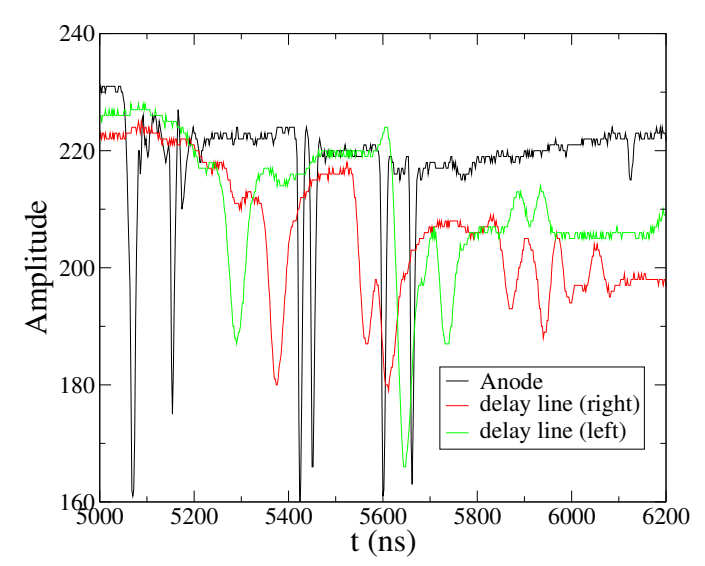

(a) Original recorded signal

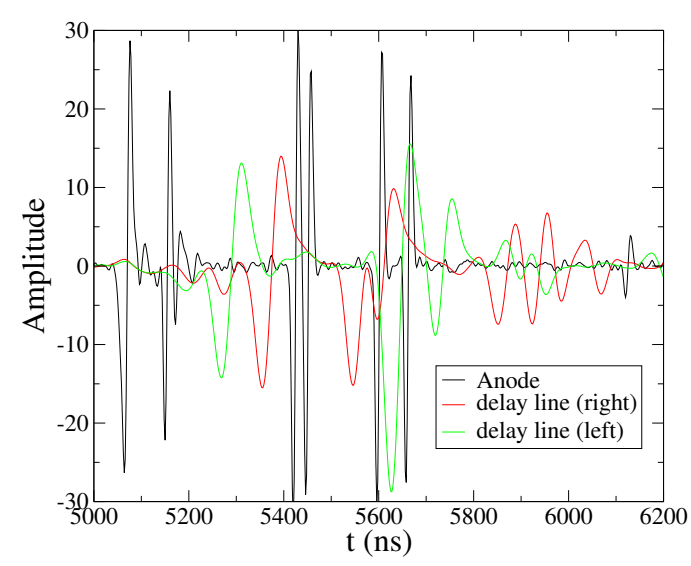

(b) After convolution with the derivative of a Hanning's filter

Figure 3: Anode signal (black) and the corresponding signals from the horizontal delay line, right (red) and left (green). The first peak is the initial muon flash.

Figure 3(a) shows the anode signal and the related signals at the ends of the horizontal delay line. The first negative peak visible in the anode signal is the initial flash, corresponding to ultrarelativistic muons travelling at a velocity very close to speed of light. This peak is used as a reference for the calibration of the time of flight. The five other peaks of high amplitude are fission events, produced by neutrons from $150 \mathrm{MeV}$ to $1 \mathrm{GeV}$. They show up also in the delay line signals. The assignment of localisation signals to the associated anode ones is based on the sum of the delays which should remain constant and equal to the total delay of the line. It can be noticed that the muon flash induces a waving of the baseline. This is treated by applying a convolution with the derivative of a Hanning's filter which is equivalent to a derivative with an attenuation of the high frequency components. The result is displayed in figure 3(b), showing that a flat baseline is restored and that the peak recognition and characterisation become much easier.

Another advantage of this numerical treatment is the shielding against parasitic signals. Figure 4 shows how the noise generated by sparkling of the detector can be suppressed and the signal enhanced, by applying the convolution which favors the frequency components of the signal. 

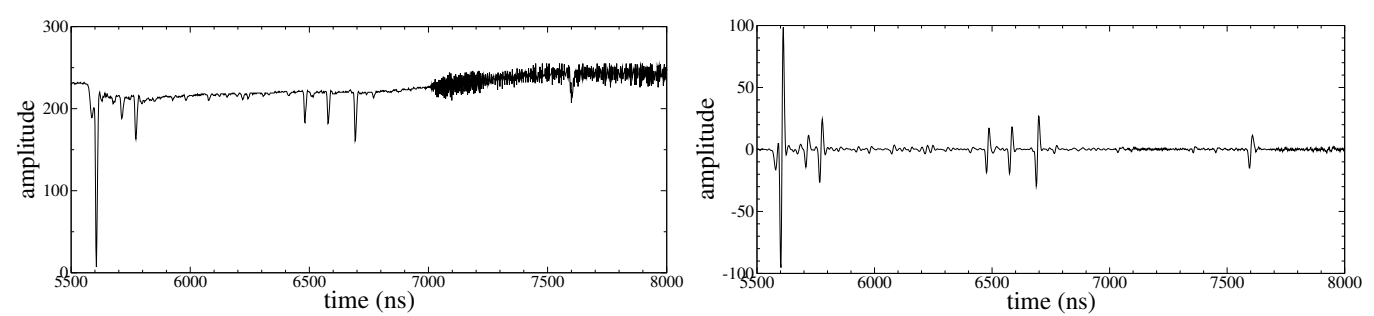

Figure 4: Left: original signal where a noise is induced by detector sparkling (beyond $7000 \mathrm{ns)}$ ). Right: when the convolution is applied the noise is suppressed whereas the signal at 7600 ns clearly shows up.

\section{Results}

The trajectory reconstruction is a specific feature of our detection system, and it is essential for the correction of the angular dependence of the efficiency. One can check its validity by looking at the reconstructed map of emitting points as shown in figure 5 in the left part. This can be compared with the density map of the target as represented in figure 5 in the right part, as obtained by scanning the local alpha radioactivity. The detailed geometry of the defect on the right side is well reproduced and the disk shape as well. However it can be noticed that the neutron flux is not uniform and it is strongly depleted in the lower region of the disk.

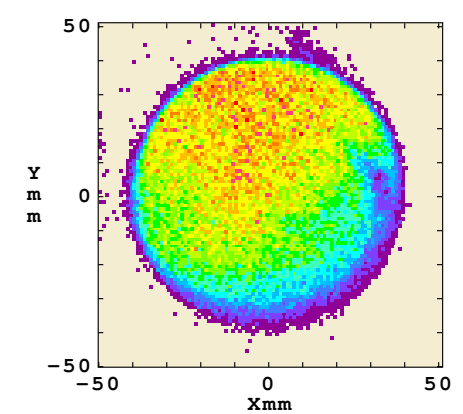

Figure 5: Left: mapping of the fission rate for a ${ }^{234} U$ target. Right: mapping of the $\alpha$-radioactivity rate for the same target.

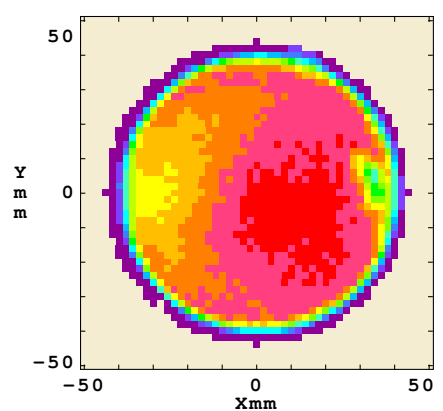

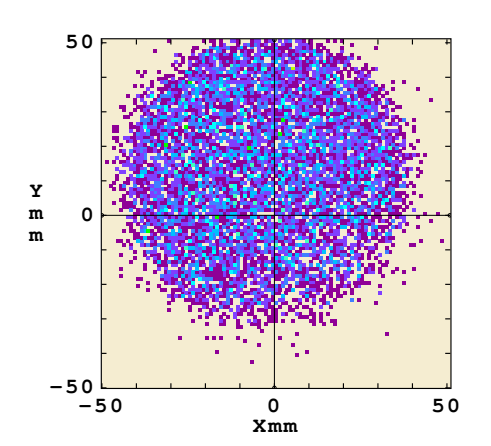

Figure 6: Mapping of the fission rate for the large bismuth target. The line crossing indicates the geometrical center of the target.

This feature is confirmed when looking at the bismuth target which extends well beyond the expected beam size, as illustrated by figure 6 . It can be clearly seen that the beam spot is shifted upward, due to geometrical misalignment. This pitfall has no harmful effect because it can be taken into account through the emitting point determination.

As already mentioned the angular efficiency drops when the fission angle increases and the setup becomes insensitive to fission occurring at angles higher than $60^{\circ}$. As a consequence the angular distribution of fission fragments for the measured isotopes should be known to calibrate the angular efficiency and to extract the cross sections. For this purpose we used the known experimental results up to $20 \mathrm{MeV}$. Beyond this energy where the measurements are very scarce we applied a constant decrease of the anisotropy, down to full isotropy at $100 \mathrm{MeV}$. 
The results presented hereafter are obtained from the fission rate referred to that of ${ }^{235} \mathrm{U}$.
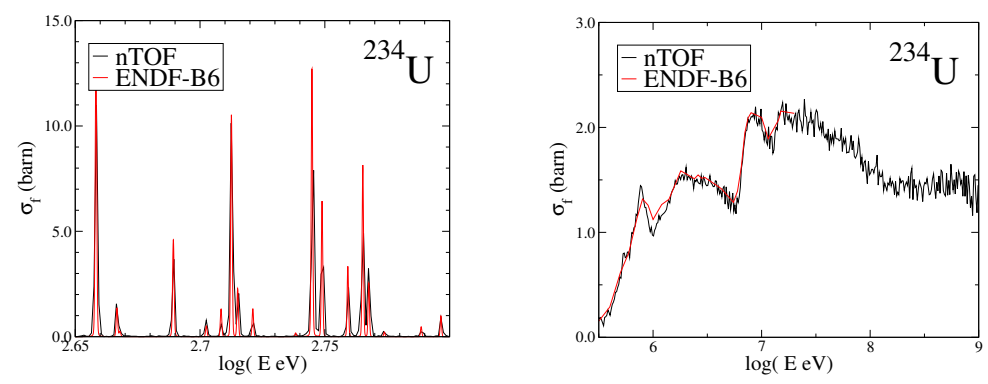

Figure 7: Fission cross section of ${ }^{234} \mathrm{U}$ compared with the evaluated data from ENDF-B6. Left: in a portion of the resonance region. Right: around and above the fission threshold.

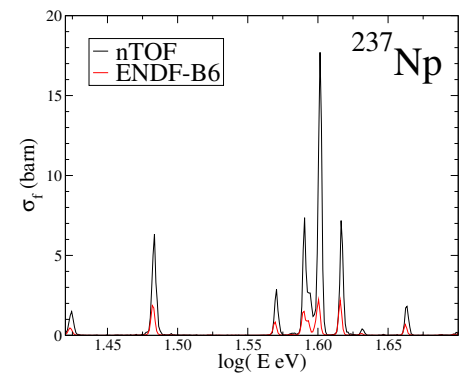

Figure 8: Fission cross section of ${ }^{237} \mathrm{~Np}$ in a portion of the resonance region compared with the evaluated ENDF-B6

Figure 7 is an illustration of the obtained results in the case of ${ }^{234} \mathrm{U}$. In the resonance region the nTOF measurement is in agreement with the ENDF-B6 data. This is also the case around the fission threshold and above. The nTOF measurement extends to much higher energies in the spallation domain where no previous measurement exists. The accuracy of the measurement is mainly driven by the uncertainty on the angular efficiency. The latter has been estimated by the dispersion of the results for different targets of the same isotope. It amounts to about $10 \%$ in standard deviation.

Figure 8 shows the case of ${ }^{237} \mathrm{~Np}$ in the resonance domain. It can be checked that the shape of resonances is well reproduced, in particular the shoulder on the resonance at $38.9 \mathrm{eV}$. However a much larger cross section is found if compared to the evaluated data. This discrepancy is specific of the resonance region, whereas a good agreement is found in the threshold region.

\section{Conclusion}

The fission cross section of actinides has been measured by detecting both fragments in coincidence. This allowed to clearly disentangle fission from other mechanisms at high energy, and the measurement covers the large energy range available at nTOF: $0.7 \mathrm{eV}$ to $1 \mathrm{GeV}$. All measurements are relative to ${ }^{235} \mathrm{U}$ and ${ }^{238} \mathrm{U}$, this requires the knowledge of their cross sections. The estimation of the angular efficiency relies on existing data which are limited generally to $20 \mathrm{MeV}$.

\section{References}

[1] Y. Kadi, White neutron sources - RB02, these proceedings

[2] A. Y. Konobeyev et al., JAERI Research Report 2002-28

[3] L. Tassan-Got et al., Fission of actinides induced by neutrons at nTOF, ND2004 conference, Santa Fe, New Mexico USA, Sept. 27- Oct. 1, 2004, AIP Conference Proceedings, Vol 769, (2005), 1529

[4] L. Ferrant, Mesures de sections efficaces de fission induite par neutrons sur les actinides du cycle du thorium à $n T O F, \mathrm{PhD}$ thesis, Université Paris-Sud, Orsay, September 7, 2005

[5] C. Paradela, Measurement of the ${ }^{234} U(n, f)$ cross section with PPAC detectors at the nTOF facility, $\mathrm{PhD}$ thesis, Santiago de Compostela, October 6, 2005 